\title{
On Relative Precedence of Coherent and Random Noise Attenuation
}

\author{
Anil Kumar Semwal ${ }^{1}$, Naresh Agarwal ${ }^{2}$ \\ ${ }^{I}$ (Processing/ Pan India Consultants, Pvt. Ltd., India) \\ ${ }_{2}^{2}$ (Processing/ Pan India Consultants, Pvt. Ltd., India)
}

\begin{abstract}
Noise attenuation has always been a challenging affair for acquisition or processing geophysicist. The quality of final product and ease for application of next main phases of processing such as deconvolution, velocity analysis, residual statics computation, stacking and migration grossly depends on the way and amount, the noise is eliminated while keeping the signal preserved. This study is aimed at analyzing and recommending the relative precedence of attenuation steps for coherent and random noises. It is inferred, using field data examples that source related coherent noise be treated first for attenuation and the random noise next in the realm of seismic data processing steps.
\end{abstract}

Keywords: Coherent noise, random noise, spectrum, stack, velocity analysis

\section{Introduction}

The seismic analyst is confronted daily with the important task of: Selecting a proper sequence of processing steps appropriate for the field data under consideration (Yilmaz, 1987). Honoring this, a choice of noise elimination subsequence, that, what is to be tackled first, coherent or random, remains with the processing geophysicist.

With this viewpoint, we opted to process a land 3D and another land 2D dataset, each on two streams. In one stream, incoherent random noise is entertained first for attenuation and coherent next, while in the other stream, coherent noise is treated first and incoherent later. Finally, we resort to recommend one stream having output with relatively better event continuity, S/N ratio and frequency components' strengths in amplitude spectrum.

One of the simplest filtering approaches used in the ground roll attenuation problems is $f-k$ filtering, which is applied in the frequency-spatial wave number domain. The $f-k$ method uses 2D Fourier transform (Embree et al., 1963; Wiggins, 1966) and the ground roll, represented by linear events with low velocities, is mapped as lines in the $f-k$ domain. It can consequently be filtered using a $2 \mathrm{D}$ band pass or reject filter. We chose $f-k$ filtering in the current study for attenuation of such noises.

Spatial prediction filtering in the frequency-offset $(f-x)$ domain is an effective method for random noise attenuation. Originally proposed by Cannales (1984), the idea exploits signal predictability in the spatial direction. The method he used to extract the predictable signal is the $(f-x)$ prediction filtering where the noise is computed by applying the prediction error filter (PEF) to the data. Soubaras, 1994, showed the inconsistency of this method, and that the correct way to proceed is to obtain the noise by applying the autodeconvolved PEF to the data. This leads to the $(f-x)$ projection filtering. We opted for $f-x$ projection filtering for random noise attenuation in this work.

\section{Method}

For the current study, we have used land 3D and land 2D seismic data sets of onshore Cambay basin, India. First of all, for both the data sets' geometry and navigation information is updated in trace headers, field statics is incorporated, a band-pass filter $(3-8-70-83 \mathrm{~Hz}) \&$ time gain function is tested and applied and first break mute is designed and applied.

The data is preprocessed in two streams. In one stream (stream-1), random noise elimination is dealt first and coherent linear noise later while in another stream (stream-2), coherent linear noise attenuation is attempted before random noise elimination.

Amplitude smoothening is carried out using SPASM (Spatial Amplitude Smoothening), random noise attenuation is achieved via application of SPARN (Signal Preserving Attenuation of Random Noise, a projective filtering technique in $f-x$ domain) and linear noise is attenuated through FKFIL ( $f-k$ filtering) in CGG-Veritas's Geocluster environment. Same values are input for various parameters of the modules used in both the streams. In this exercise, results and methodology for 3D dataset are more elaborated than that of $2 \mathrm{D}$ dataset using illustrations. 


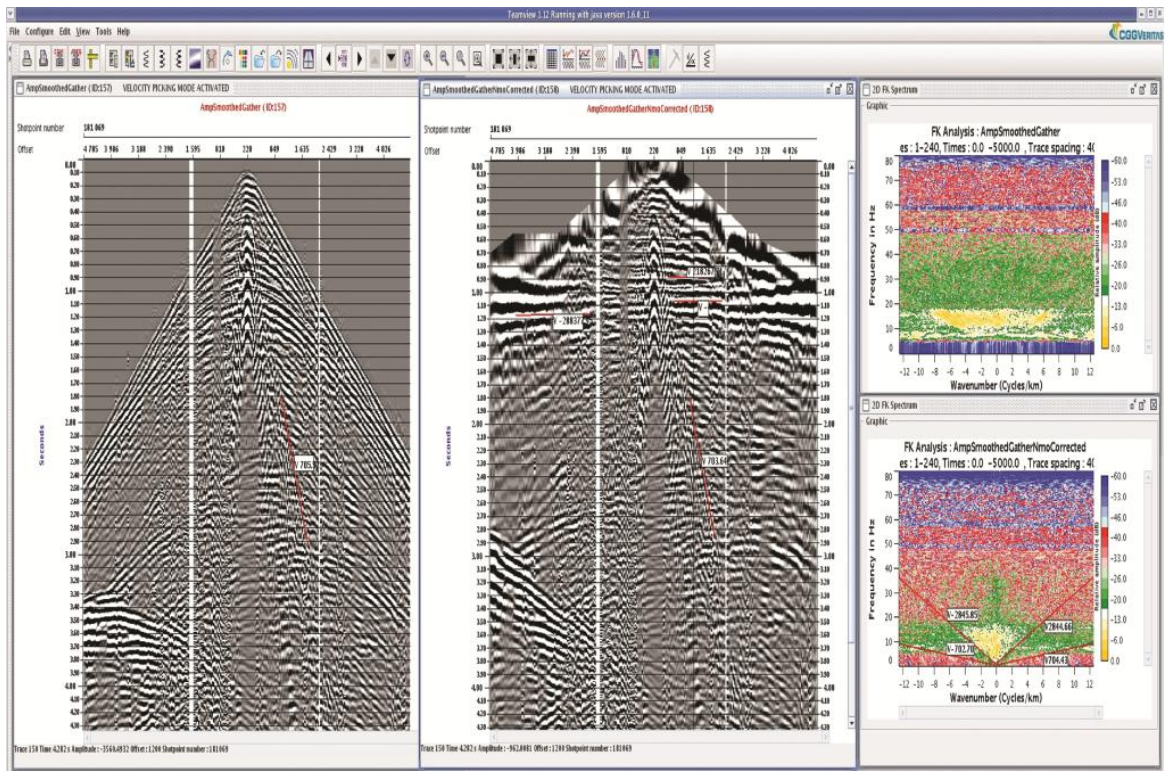

Figure 1. Land 3D: Original Shot gather (left), It's NMO corrected version (middle) and their respective f-k spectra (top right and bottom right)

Application of f-k filtering, requires gathers to be NMO corrected, so that, hyperbolic events in the gathers try to flatten themselves, whereas, linear noise is least affected. It is observed, that after application of NMO correction, linear noise remains undistorted with the same velocity (e.g. in figure 1, a land 3D scenario is illustrated, where a linear event is mapped with same velocity $\sim 703 \mathrm{~m} / \mathrm{s}$ on both versions of a shot gather), whereas, hyperbolic events have straightened themselves with very high velocities (> $200000 \mathrm{~m} / \mathrm{s}$ and up to $\infty$ ) particularly in the time zone 900-1300 ms. Figure 1 testimony this, wherein, velocities of some of the hyperbolic events and linear events are depicted. Figure 1 comprises of four panels, the original gather, its NMO corrected version and corresponding f-k spectra.

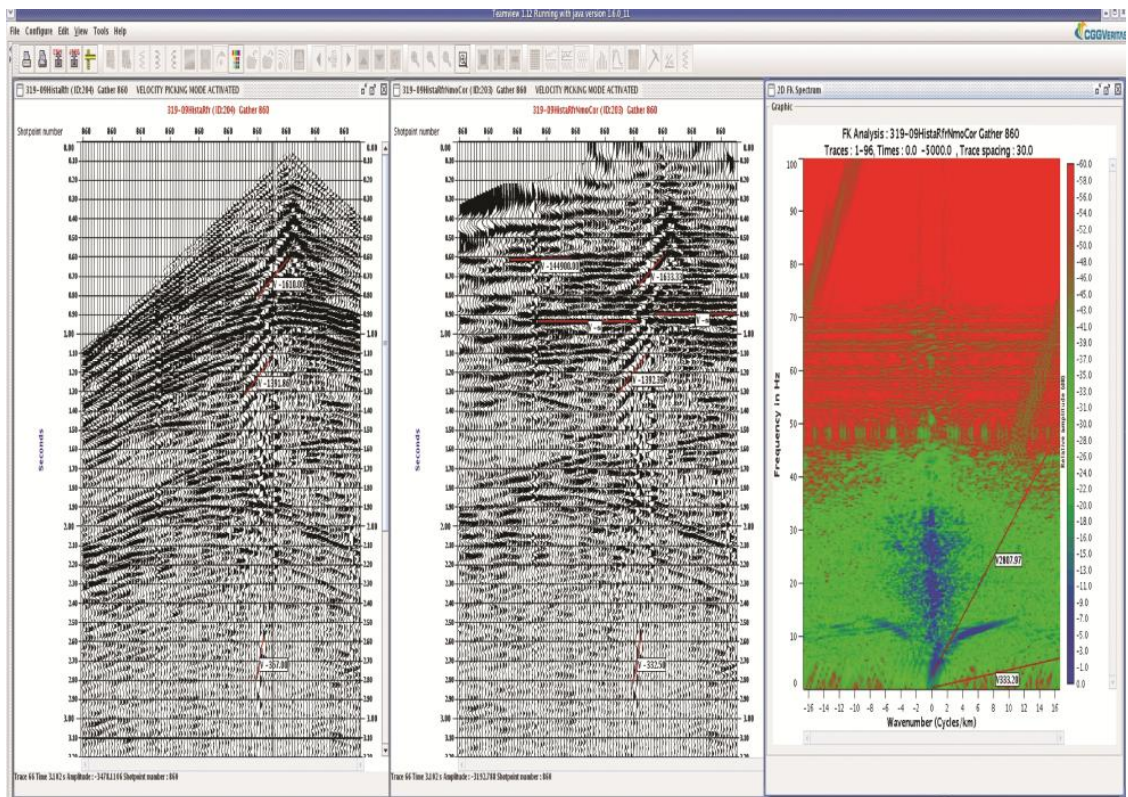

Figure 2. Land 2D: Original Shot gather (left), it's NMO corrected version (middle) and f-k spectra of NMO corrected gather (right)

In the f-k spectrum panel of NMO corrected data, hyperbolic events show up with very high velocities and are mapped around frequency axis near $\mathrm{k}=0$ while the rest of the phenomena such as high amplitude, low velocity, low frequency dispersive coherent noise etc. maps away of frequency axis on higher wave numbers in both the quadrants (figure 1). Same analysis can be drawn for 2D case and is illustrated in figure 2.

For 3D and 2D datasets, a symmetrical reject fan filter each with velocities $704 \mathrm{~m} / \mathrm{s}$ to $2844 \mathrm{~m} / \mathrm{s}$ and 
$333 \mathrm{~m} / \mathrm{s}$ to $2808 \mathrm{~m} / \mathrm{s}$ respectively is designed and applied with a taper and an anti-aliasing filter on NMO corrected gathers in $f-k$ filtering process. Finally, inverse NMO correction is applied on the gathers, after coherent linear noise is eliminated using $f$ - $k$ filtering.

For 3D and 2D datasets, representative input shot gather, stream-1 processed gather, stream-2 processed gather and corresponding amplitude spectra are shown in figure $3 \&$ figure 4 respectively.

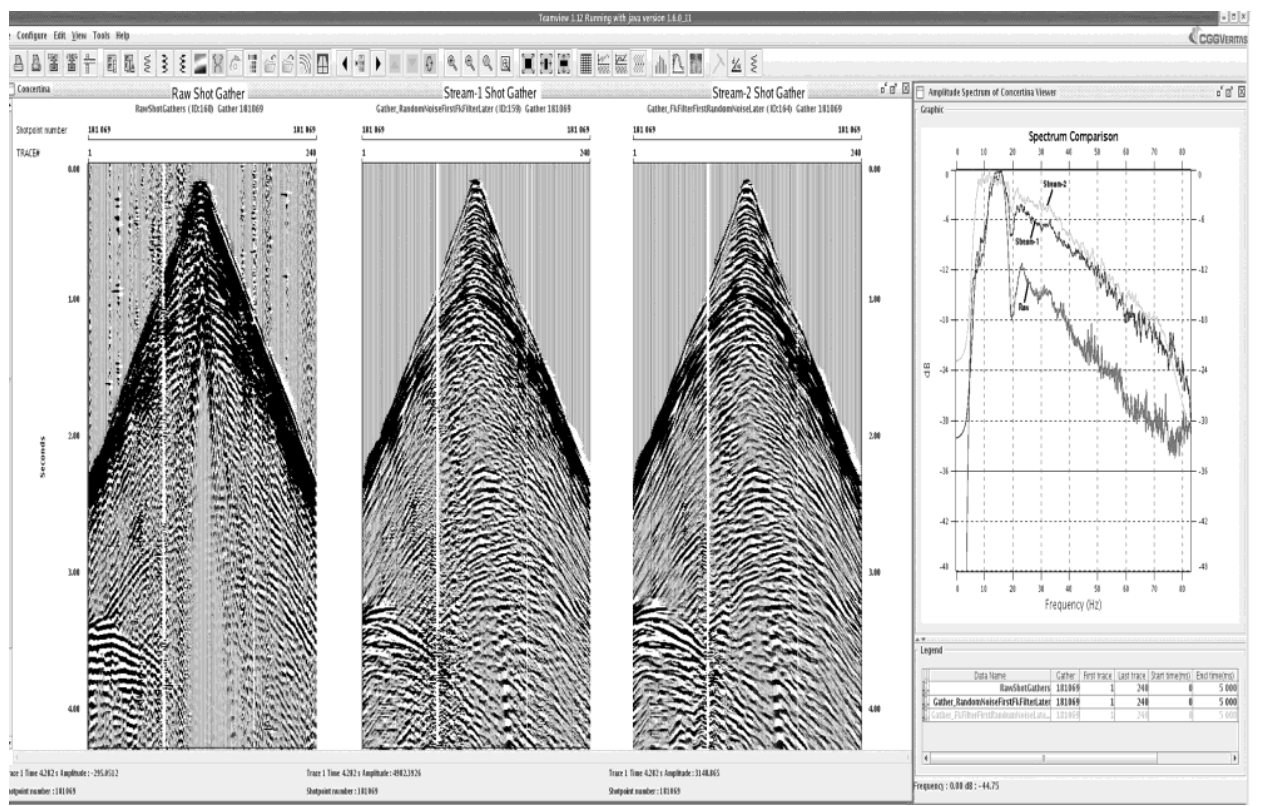

Figure 3. Land 3D: Original Shot gather (left), Stream-1 and Stream-2 gather (middle) and their respective amplitude spectra (right)

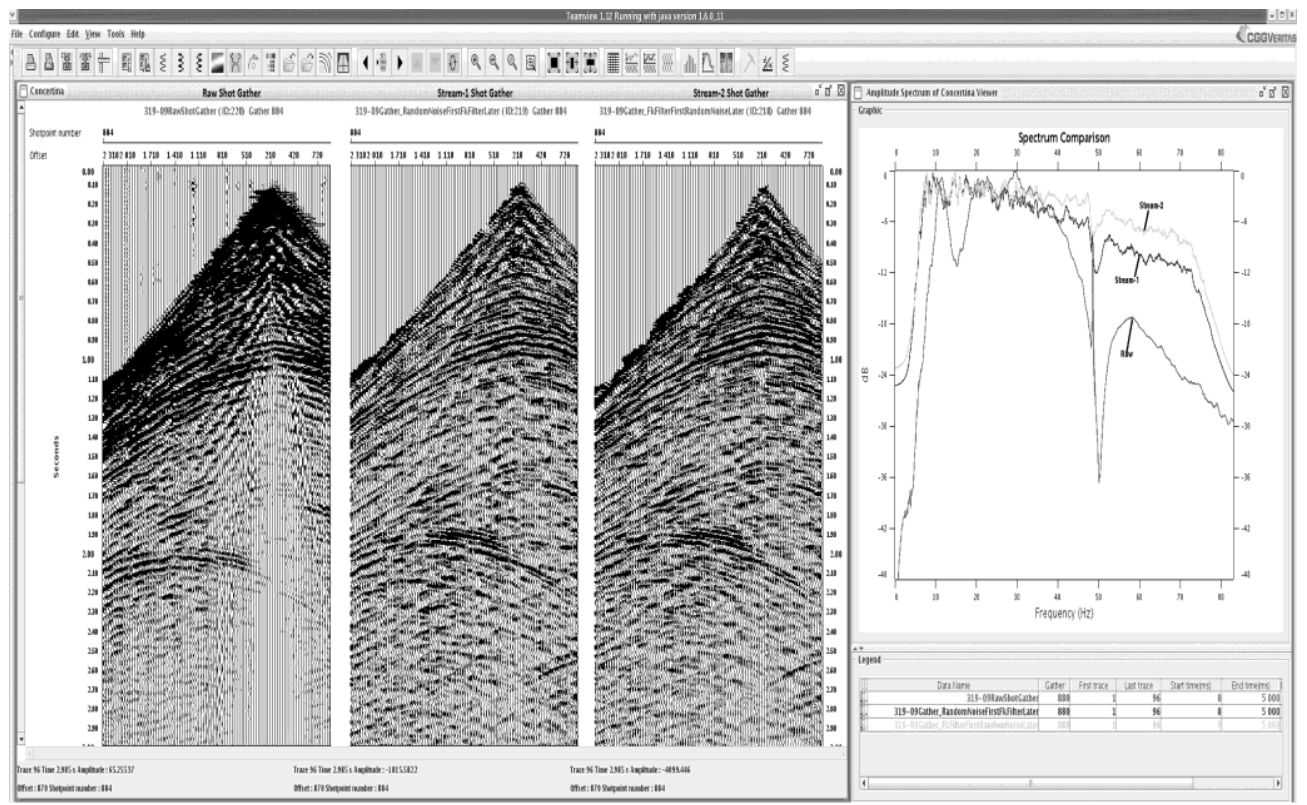

Figure 4. Land 2D: Original Shot gather (left), Stream-1 and Stream-2 gather (middle) and their respective amplitude spectra (right)

After noise is eliminated, deconvolution parameters are estimated using auto correlation function (ACF) and tested for operator length $(\mathrm{OL})$, prediction distance $(\mathrm{PD})$ and white noise $(\mathrm{WN})$. A two window deconvolution is chosen in time zones $0-1400 \mathrm{~ms}$ and $1200-5000 \mathrm{~ms}$ in the current work. Auto correlation function and subsequent parameter testing suggests an OL $(=240 \mathrm{~ms})$ and $\mathrm{PD}(=8 \mathrm{~ms}, 14 \mathrm{~ms})$ for the above said temporal windows. ACF for 3D dataset only is shown in figure 5. Testing for white noise is carried out and a $0.1 \%$ is found to be optimum. Test displays for both datasets and ACF, velocity analysis for 2D dataset are not shown here in view of avoiding too many illustrations. 


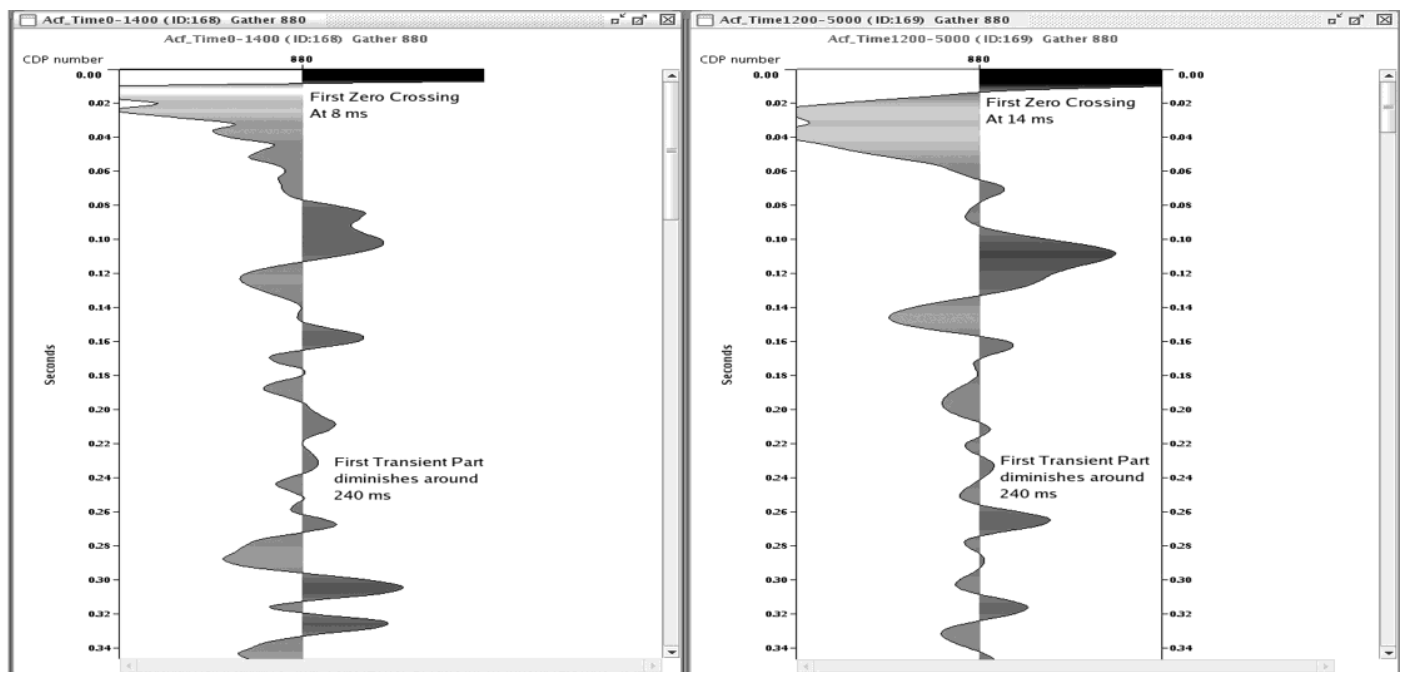

Figure 5. Land 3D: Auto Correlation Function (ACF) for two time windows: 0-1400 ms. (left) and 1200- 5000 ms. (right)

Now deconvolution velocity analysis is carried out at every $50^{\text {th }} \mathrm{CDP}$ (at an interval of $1 \mathrm{Km}$ ) in both the streams and is illustrated in figure 6 for 3D dataset only. In figure 6 , left $\&$ right frames correspond to stream- 1 and stream-2 respectively.

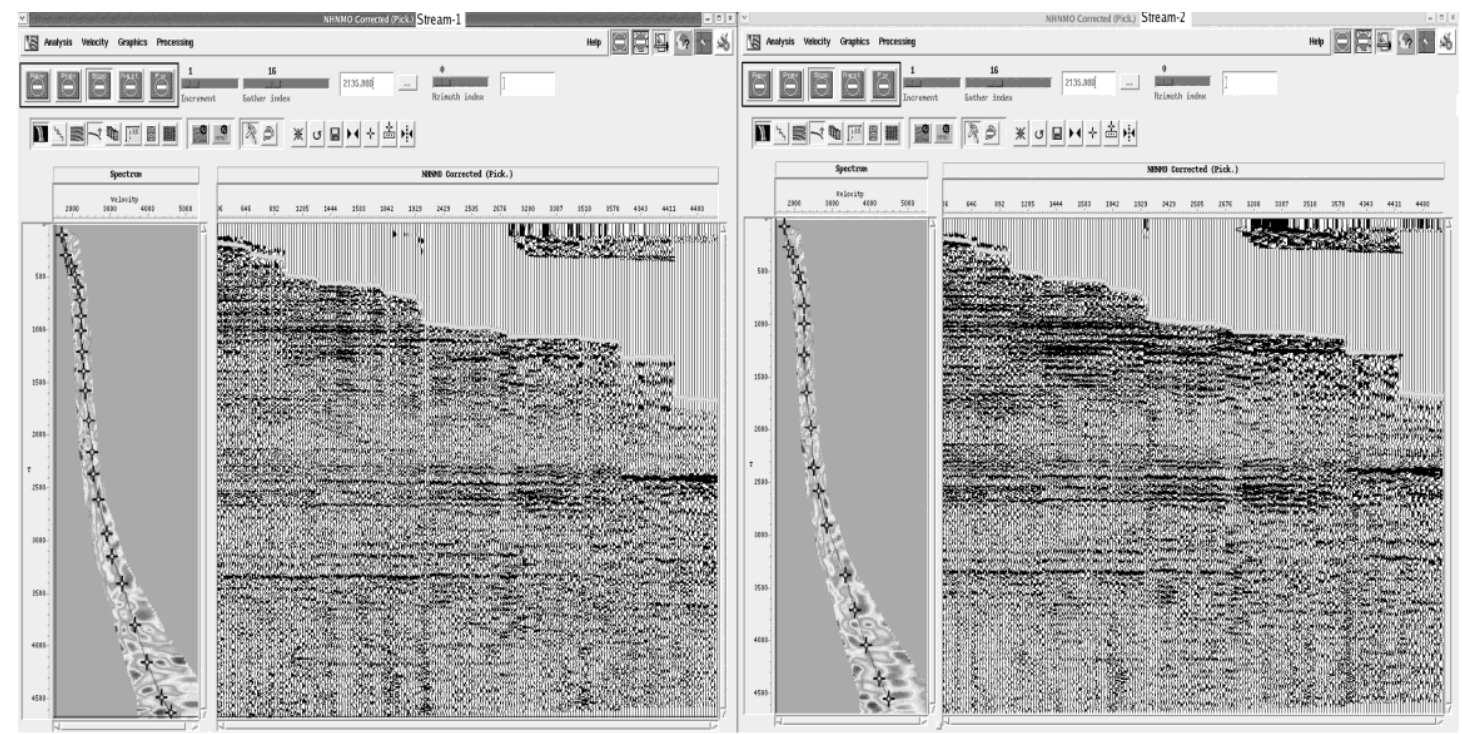

Figure 6. Land 3D: Velocity picking on stream-1 data (left) and on stream-2 data (right)

\subsection{Land 3D}

\section{Results and Discussion}

Figure 3 illustrates that coherent linear noise is almost washed away in both the processing streams (although more effectively in stream-2 gather) and hitherto noise masked hyperbolic signals, now show up with good stand out particularly in near to intermediate offset zone. Amplitude spectra also suggests that there is a continuous boosting of frequency components up to $75 \mathrm{~Hz}$ from raw input, stream-1 to stream-2 gather. Stream2 gather is sharper with lesser noise contamination and relatively better event continuity than stream-1 gather.

It can be observed from velocity analysis frames of figure 6, that events' continuity and signal to noise ratio is better in stream-2 than that for stream-1. Using these picked velocities, stacks after deconvolution are produced for both the streams (figure 7), which shows that better continuity and higher $\mathrm{S} / \mathrm{N}$ ratio can be attributed to stream-2 processed data than stream-1. 


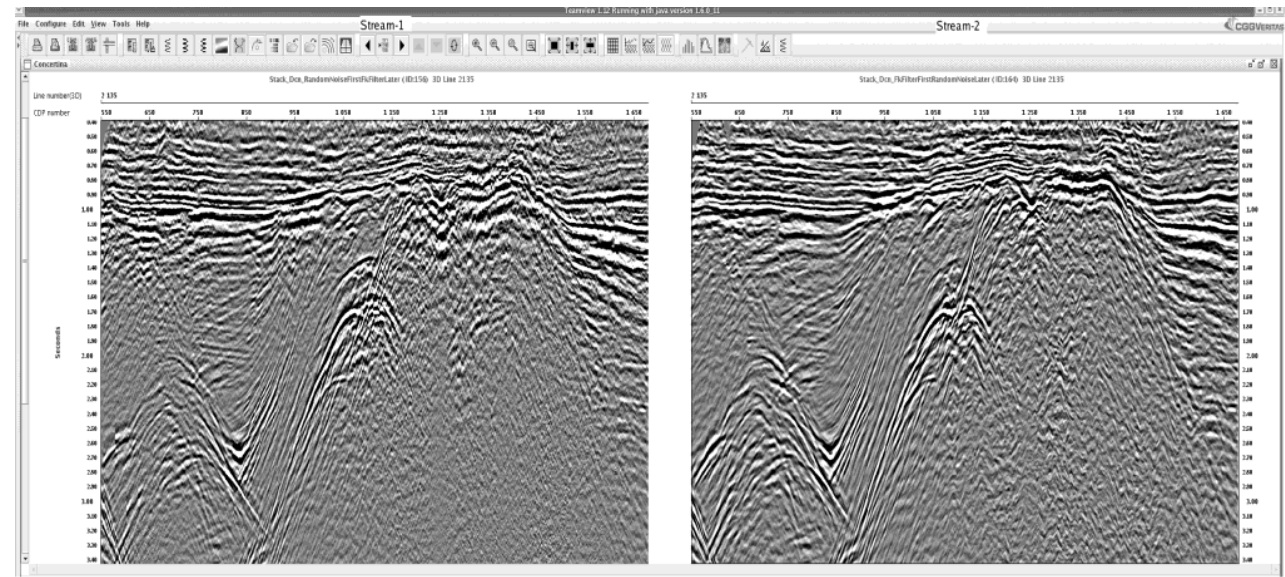

Figure 7. Land 3D: Stack after deconvolution for stream-1 (left) and stream-2 (right)

Figure 8 comprises of brute stack \& amplitude spectra of various stream-2 stacks produced in this exercise for analysis purposes of $3 \mathrm{D}$ dataset.

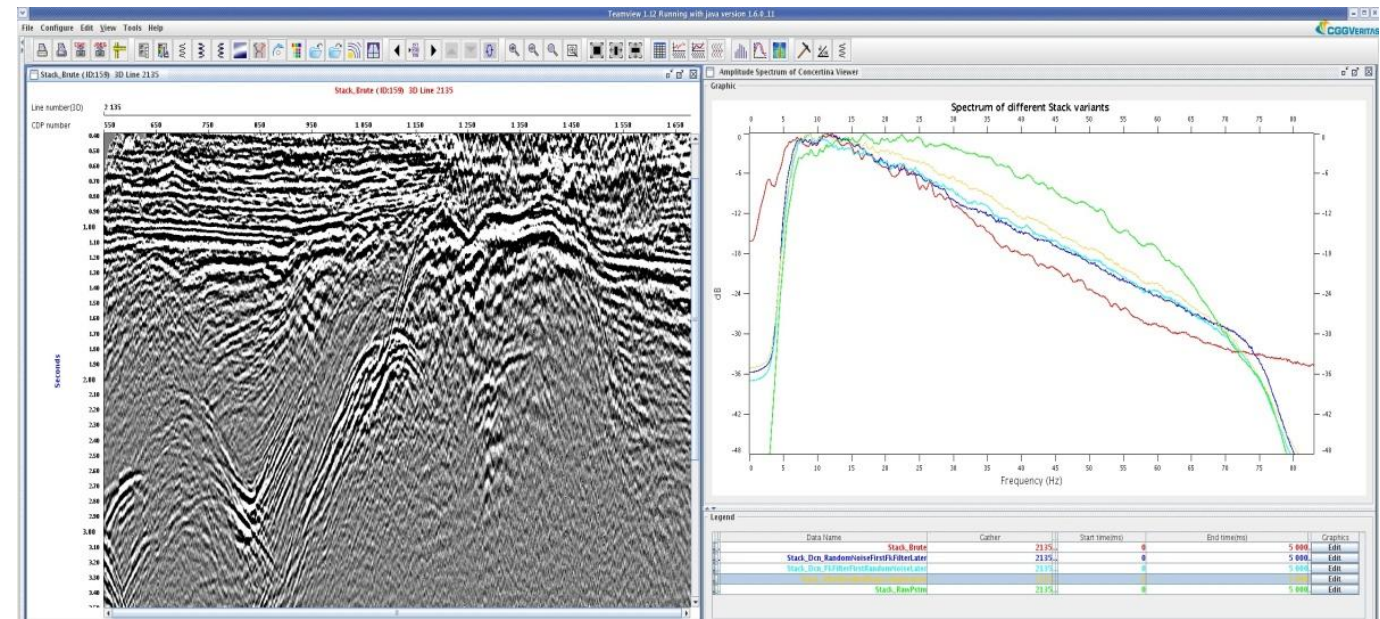

Figure 8. Land 3D: Brute stack for an inline (left) and spectra of stacks corresponding to various subsequent processes from brute to PSTM (right)

Next, residual statics computation and prestack time migration (PSTM) are carried out on stream-2 and corresponding stacks for 3D dataset are illustrated in figure 9.

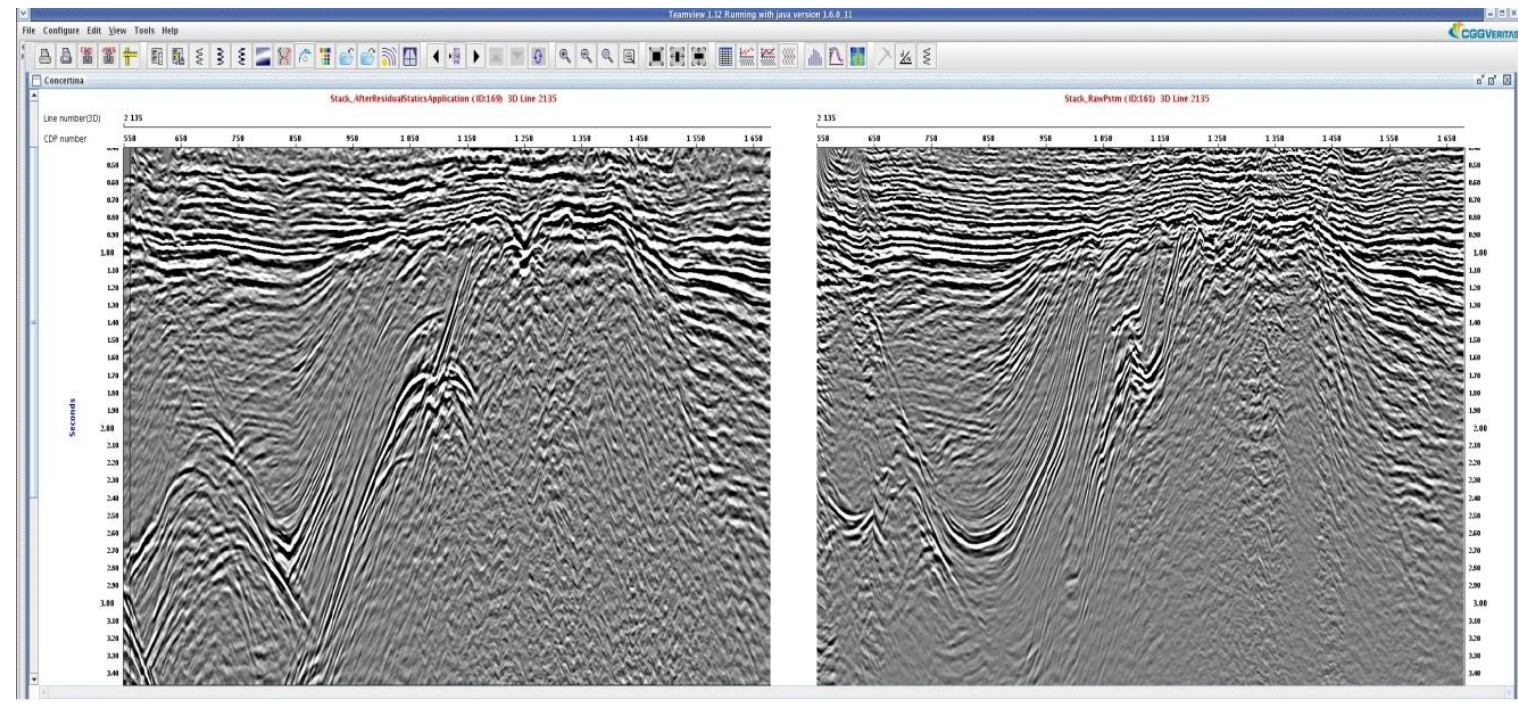

Figure 9. Land 3D: Stack after residual statics application (left) and raw PSTM stack (right) 
Land 3D case for stream-2 generated data is dealt further and basic processing cycle up to PSTM is carried out to show the quality of the final product through raw PSTM stack. (figure 9). It can be in inferred from spectra shown in figure 8 , that data is continuously gaining strength and acquiring more and more flatness particularly in the frequency band $12-70 \mathrm{~Hz}$ during its journey from brute stack (in red), stream-1 stack after deconvolution (in violet), stream-2 stack after deconvolution (in blue), stack after application of residual statics (in orange) to raw PSTM stack (in green).

\subsection{Land 2D}

Stream-2 gather shows superiority over stream-1 gather in terms of events' continuity, $\mathrm{S} / \mathrm{N}$ ratio and overall amplitude spectrum strength up to $83 \mathrm{~Hz}$ (Figure 4).

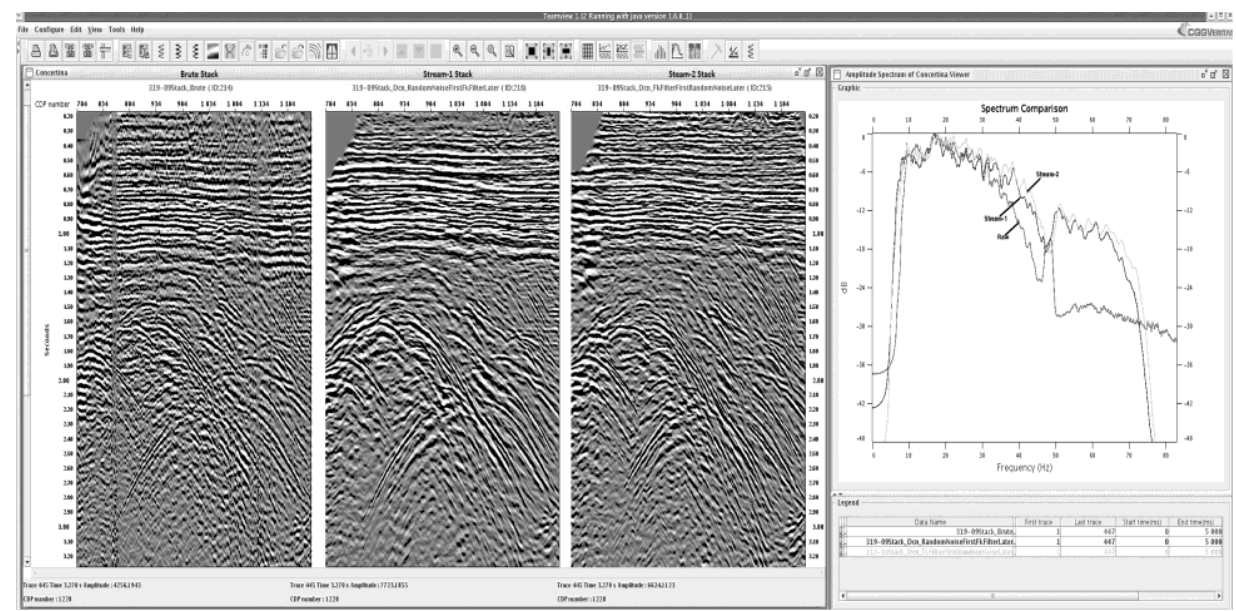

Figure 10. Land 2D: Brute stack (left), Stack after deconvolution for stream-1 and stream-2 (middle) and respective amplitude spectra (right)

Figure 10 shows brute stack, stacks after deconvolution for approaches based on stream-1 \& stream-2 with corresponding amplitude spectra for $2 \mathrm{D}$ dataset. It also supports that shallow events stand out with better continuity and contains relatively more robust frequency components in case of stream-2 stack section than that of stream-1.

\section{Conclusion}

Keep in mind that the success of a process depends not only on the proper choice of parameters pertinent to that particular process, but also on the effectiveness of the previous processing steps (Yilmaz, 2001). With this as a central point, we bifurcated, the problem of noise attenuation in the pre-deconvolution stage in two streams, to analyze their relative effectiveness on the output seismic gathers and stacks. Standard noise attenuation techniques are implemented and same values of parameters during processing and output displays are used in both the streams.

Coherent noises are predictable and if we start with random noise attenuation prior to coherent noise attenuation, the inherent predictability of coherent noise gets perturbed and leads to weakening of the attenuation algorithm's effectiveness.

The above results on gathers, stack variants and corresponding spectra for land 3D and land 2D datasets support that in the realm of noise attenuation, coherent noise should be entertained first and the random later.

\section{Acknowledgments}

We thank the management of Pan India Consultants, Pvt. Ltd. to facilitate and inspire us during this work.

\section{References}

[1] Yilmaz Ozdogan, Seismic Data Analysis, (SEG, 2011) 283-284, 289-290.

[2] Cannales, L., 1984, Random noise reduction: 54th Annual International Meeting, SEG, Expanded Abstracts, $525-527$.

[3] Embree, P., Burg, J. P. and Backus, M. M., 1963, Wide-band velocity filtering - the pie-slice process: Geophysics, 28, 948-974.

[4] Soubaras, R., 1994, Signal-preserving random noise attenuation by the f-x projection: Expanded Abstracts of the 64th Annual SEG Meeting, 1576-1579.

[5] Wiggins, R. A., 1966, W-K Filter design: Geophysical Prospecting, 14, 427-440.

[6] Yilmaz Ozdogan, 1987, Seismic Data Processing, Published by SEG, xi.

[7] Yilmaz Ozdogan, 2001, Seismic Data Analysis, Published by SEG, 91. 